\title{
Advancing understandings on students' mobility as a tool to reach 2030 Agenda
}

\section{Rosalina Pisco Costa}

Department of Sociology and CICS.NOVA.UÉvora, Universidade de Évora, Portugal.

\begin{abstract}
This paper interrogates the role of students' mobility within the framework of 2030 Agenda, specifically, the prospects of reaching Sustainable Development Goal 4, a quality education for all by 2030. Empirical data draw upon a report that analyzes mobility's figures over the last five academic years at the University of Évora, Portugal. Important insight can be gained from 2030 Agenda when looking at the strong imbalance observed between IN and OUT mobility, countries of origin and destination, gender and age distribution of the students' missions. The paper claims for a call for action in order to use academic mobility as a tool to meet the 2030 Agenda for Sustainable Development. While academic mobility can and should be understood as part of a broader strategy aiming the dissemination and internationalization of knowledge and skills, acquisition and transfer of good practices, it should not neglect a commitment that through SDGs unites us all: to reduce inequalities and to improve the quality of life among individuals around the world.
\end{abstract}

Keywords: Students' Mobility; Higher Education; Portugal; Sustainable Development Goals; 2030 Agenda. 


\section{Introduction}

This paper interrogates students' mobility as a tool to meet the goals set by the 2030 Agenda for Sustainable Development, specifically Sustainable Development Goal 4, which calls for a quality education for all by 2030 .

International student mobility is a key for understanding how students become mobile within their present educational and future working lives (Cairns, 2014). Moreover, international organizations such as UNESCO and OECD are unanimous in recognizing the importance of international mobility of students as part of a quality education goal (OECD 2019; UNESCO 2019). As the United Nations underlines, "the 2030 Agenda for Sustainable Development, adopted by all United Nations Member States in 2015, provides a shared blueprint for peace and prosperity for people and the planet, now and into the future" (UN, 2020). According to the same source, the 17 Sustainable Development Goals (SDGs) constitute an urgent call for action by all countries, interacting in a global partnership. Working for sustainable development isn't about being focused on economic growth, rather to spur it while improving the life conditions of individuals, ending poverty and other deprivations, reducing inequalities, improving health and education, and, at the same time, tackling climate change and working to preserve oceans and forests.

Having these assumptions as a backdrop, this paper summarizes some conclusions drawn upon an internal report that analyses recent mobility data at the University of Évora, Portugal. While the report comprehends a broad analysis of the data available for the academic years between 2014/15 and 2018/19, with regard to both student and staff mobility, this paper focuses only in the students' mobility.

Despite confirming much of what we already knew, the following findings also shed light on a set of aspects that deserve close and deep reflection. Academic mobility is not just an episodic event in the life of the individuals without further consequences. Securing an exchange abroad is part of improving the quality students experience in higher education (QS 2019). Academic mobility can and should be understood as part of a broader strategy for the dissemination and internationalization of knowledge and skills, acquisition and transfer of good practices. At the same time, it should not neglect a commitment that through SDGs unites us all: to reduce inequalities and to improve the quality of life among individuals around the world (Independent Group of Scientists appointed by the SecretaryGeneral, 2019).

\section{Data and Methods}

According to official data, a total of 1877 students' mobility missions were registered in the last five academic years at the University of Évora. 
The University of Évora is a small scale university located in Évora, a medium-sized city in the south of Portugal. The University was established on the initiative of the Crown, under a Jesuit management in 1559. Following the Jesuits' expulsion in 1759, the University was closed, without, however, being extinguished. It was restored, two centuries later, in 1973, under the name "Instituto Universitário de Évora", and at the end of the 70s, the original designation of the University was recovered. During the 80s and the 90s, the University has consolidated itself in its teaching and scientific activity and gained its administrative and financial autonomy. The first decade of 2000 was marked by transformative events that were instrumental in the current structural, teaching and scientific setting of the University of Évora. By now, the University comprises four thematic core Schools (School of Social Sciences, School of Science and Technology, School of Arts and the School of Nursing), plus a doctoral school, the Institute for Research and Advanced Training, which aggregates several research centres and Chairs. Currently, the University counts more than 7000 students in bachelor, master and PhD courses (UE, 2019).

The information provided by the University services was compiled by the author into a single database and completed whenever possible using data confrontation and triangulation (e.g. between country of origin and nationality, study cycles and organic units, program and duration, etc.). The analysis was carried out using IBM SPSS Statistics software (version 24, UÉvora licence). All data were anonymized and worked on in compliance with the ethical and deontological principles that guide the good practices of scientific research, namely by adherence to the International Sociological Association Code of Ethics (ISA, 2001).

\section{Findings}

\subsection{Balance In/Out}

In total, 1877 students' mobility missions were registered in the last five academic years at the University of Évora. In the period considered, incoming mobility (IN) (total of 1311, annual average of 262.2 missions) almost doubles outgoing mobility (OUT) (total of 566, annual average of 113.2 missions), with a difference of more than 149 missions on average, favouring IN mobility. Despite the growing trend of recent years, the proportion in 2018/19 is still approaching 70/30 (Figure 1). 


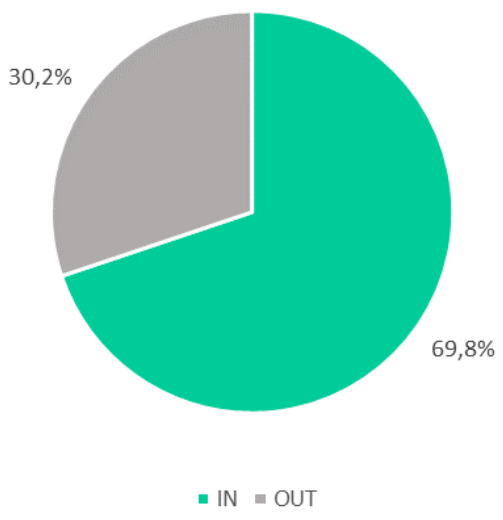

Figure 1. Students' mobility missions by type (2014/15-2018/19). Source: Own elaboration.

\subsection{Origin and destination}

Compared to IN mobility missions, the number of countries involved in OUT mobility decreases to about half (40 vs. 23). Consequently, also decreases the geographical and cultural reach of such a type of mobility. Data shows the relatively recent attractiveness of countries in eastern, central and south-eastern Europe such as Poland, the Czech Republic, the Slovak Republic and Bulgaria, which come to compete with the so-called traditional destinations, based on the relations of linguistic, geographical or historical proximity between countries, such as Spain, Italy or Brazil. A set of fewer numerous missions, but nonetheless, quite iconic due to the cultural specificity or geographic distance of the destinations they contemplate, is explained by the existence of specific programs aimed at expanding mobility opportunities in institutions from non-European partner countries. However, OUT mobility aiming those countries is scarce (e.g. Malaysia, Thailand, Turkey, Vietnam) or even non-existent (e.g. Ecuador, Philippines, India, Laos, Mongolia, Myanmar, Nepal, Pakistan, Tibet, East Timor) (Figure 2 and Figure 3). 


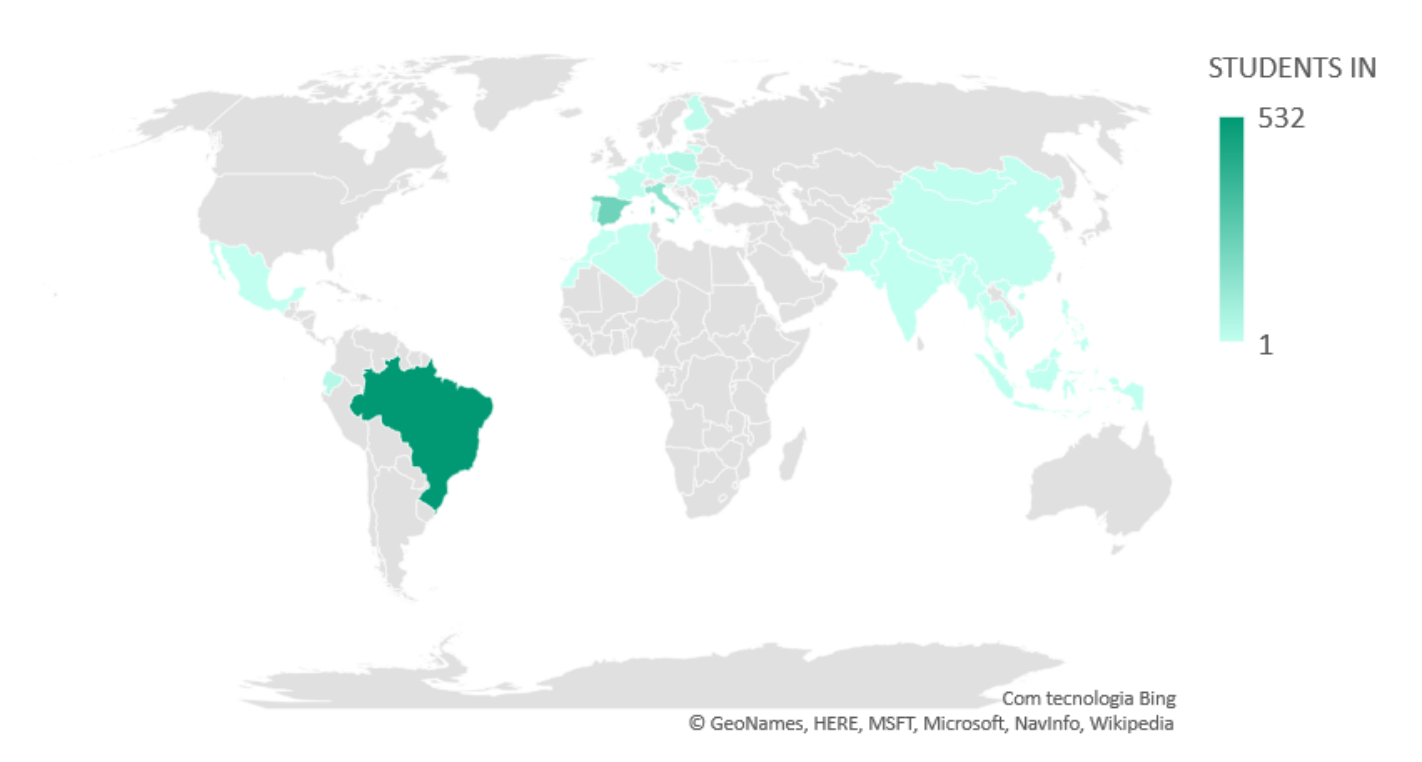

Figure 2. Countries of origin involved in students' mobility missions (2014/15-2018/19). Source: Own elaboration. $n=1311$

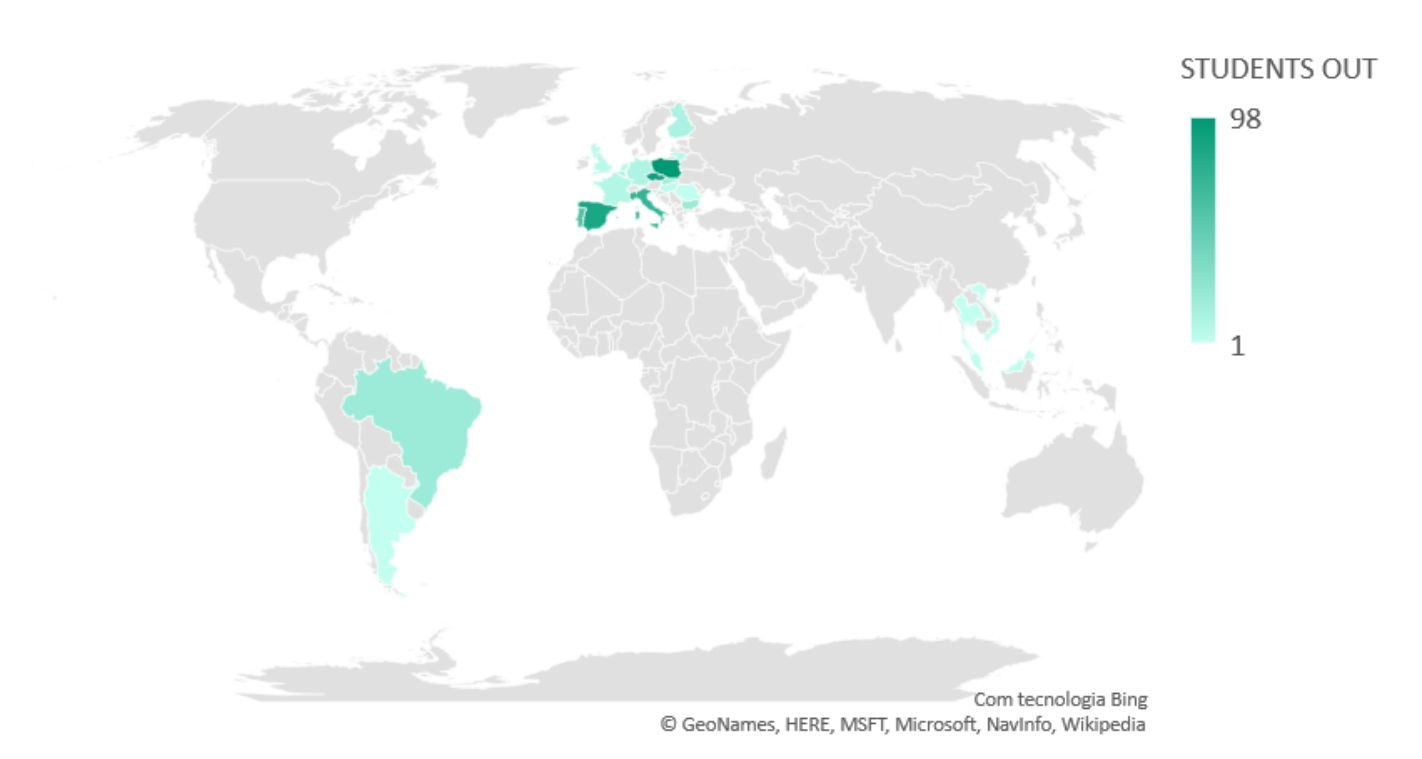

Figure 3. Countries of destination involved in students' mobility missions (2014/15-2018/19). Source: Own elaboration. $n=566$ 


\subsection{Age}

The age at which students carry out the mobility experience is very similar in both IN and OUT mobility missions. Nonetheless, when analysed by degree, age differences assume greater visibility. The average age of students increases with the progression through the study cycle also increases. In other words, the average ages are younger, in ascending order, in bachelor, master and $\mathrm{PhD}$ degrees. When comparing the average ages of IN and OUT mobility missions, data shows that bachelor students experiencing IN mobility are older than the OUT (average age 23.69 and 22.55 years, respectively). This difference also corresponds to a greater range of ages in the case of IN mobility, ranging from 19 to 59 years old in the case of undergraduate degrees, whereas students experiencing OUT mobility do it later and for a smaller period of time: between 20 and 34 years old. It is also observed that the Masters and $\mathrm{PhD}$ students experiencing OUT mobility are older than those experiencing IN mobility (mean age 29.65 and 40 years and 25.44 and 31.95, respectively) (Table 1).

Table 1. Statistics for students' age by type of mobility and degree

\begin{tabular}{|l|l|r|r|r|r|r|r|}
\hline \multirow{2}{*}{\multicolumn{2}{c|}{}} & \multicolumn{3}{c|}{ IN } & \multicolumn{3}{c|}{ OUT } \\
\cline { 2 - 8 } & Valid & BSc. & MSc. & Ph.D. & BSc. & MSc. & Ph.D. \\
\cline { 2 - 8 } & Missing & 935 & 107 & 39 & 325 & 31 & 2 \\
\hline Mean & 23,69 & 25,44 & 31,95 & 22,55 & 29,65 & 40,00 \\
\hline Mode & 22 & 25 & 29 & 21 & 25 & $28^{a}$ \\
\hline Standard Deviation & 3,550 & 2,963 & 7,736 & 2,213 & 6,275 & 16,971 \\
\hline Minimum & 25 & 21 & 25 & 20 & 22 & 28 \\
\hline Maximum & 25 & 59 & 46 & 55 & 34 & 46 & 52 \\
\hline Percentile & 50 & 23,00 & 25,00 & 29,00 & 22,00 & 28,00 & 40,00 \\
\cline { 2 - 8 } & 75 & 25,00 & 26,00 & 35,00 & 23,00 & 32,00 & 28,00 \\
\hline
\end{tabular}

a. There are several modes. The lowest value is shown.

\subsection{Gender}

Altogether, data show a feminization of student mobility, with $62.8 \%$ of the missions being carried out by women and $37.2 \%$ by men (Figure 4 ). 


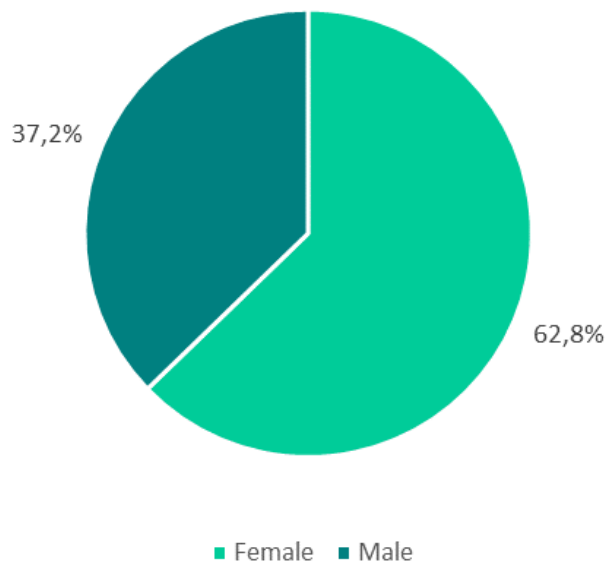

Figure 4. Students' mobility missions by gender (2014/15-2018/19). Source: Own elaboration.

A detailed analysis by gender reveals that there is a greater proportion of OUT mobility in men than in women, namely $34.3 \%$ against $27.7 \%$ (Figure 5).

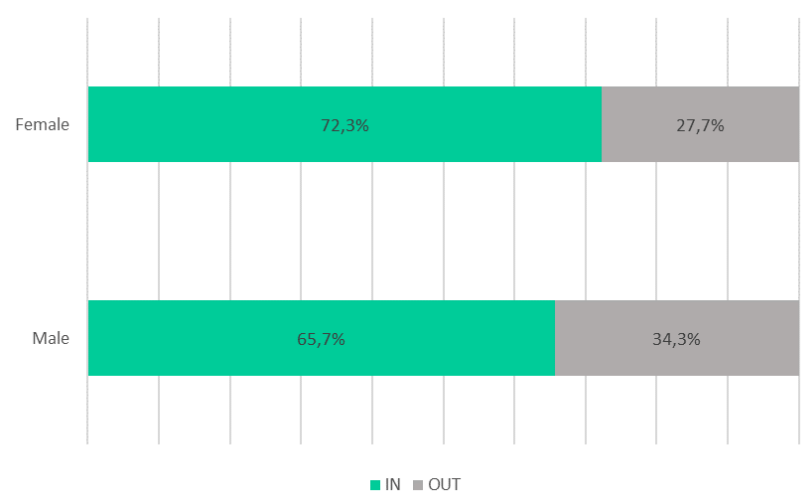

Figure 5. Students' mobility missions by gender and type (2014/15-2018/19). Source: Own elaboration..

\section{Conclusions}

In this paper, students' mobility was analysed within the prospects of Sustainable Development Goal 4, a quality education for all by 2030. Important insight can be gained from 2030 Agenda when looking at the strong imbalance observed between IN and OUT mobility, countries of origin and destination figures, as well as across gender and age. While nothing can be done to change the past, a call for action is needed to change the 
future. Moreover, there is also an ethical commitment which entails us all for sustainable development in higher-education institutions (Freitas et al., 2019).

There is a need to increase and diversify awareness actions in order to find manyfold candidates able to engage in mobility missions abroad, despite gender and age. Using a non-gendered and non-aged biased language appears to be crucial in reaching a more diverse audience. Additionally, it is important to increase awareness for students interested in an outgoing mobility experience focusing on the diversity of countries and cultures. Doubtless, academic mobility can be a tool to meet the 2030 Agenda for Sustainable Development, yet we have to move more assertively and faster, otherwise we will fail to meet the SDGs targets.

\section{References}

Cairns, D. (2014). Youth Transitions, International Student Mobility and Spatial Reflexivity. Being Mobile? London: Palgrave Macmillan.

Freitas, A.C., Quaresma, P., Balbino, A.C., Costa, Rosalina, Secca Ruivo, I., Rato, L. M., \& Calado, J. G. (2019). Ethical Governance for Sustainable Development in Higher Education Institutions. Lessons from a Small Scale University. In S.R. Nair \& J.M.SaizAlvarez (Ed.), Handbook of Research on Ethics, Entrepreneurship, and Governance in Higher Education (pp. 214-237). Hershey: IGI Global. doi: http://dx.doi.org/10.4018/978-1-5225-5837-8.ch010

Independent Group of Scientists appointed by the Secretary-General (2019). Global Sustainable Development Report 2019: The Future is Now - Science for Achieving Sustainable Development. New York: United Nations.

ISA. (2001). Code of Ethics of the International Sociological Association. Madrid: ISA. Retrieved from http://www.isa-sociology.org/about/isa_code_of_ethics.htm. Accessed January 23, 2020.

OECD (2019). Education at a Glance 2019: OECD Indicators, Paris: OECD.

QS (2019). Erasmus Without Paper - How to Make it Happen. Free white paper. MoveON. Retrieved from https://www.qs.com. Accessed January 23, 2020.

UE. (2019). Relatório de Atividades - Universidade de Évora 2018 [Report of Activities 2018]. Évora: Universidade de Évora.

UN (2020). Sustainable Development Goals Knowledge Platform. Retrieved from https://sustainabledevelopment.un.org/. Accessed January 23, 2020.

UNESCO (2019). SDG 4 Data Digest. How to Produce and Use the Global and Thematic Education Indicators. Montreal: UNESCO Institute for Statistics. 